\title{
Soluble Urokinase Plasminogen Activator Receptor and the Risk of Coronary Artery Disease in Young Chinese Patients
}

\author{
Yuli Huang, ${ }^{1}$ Haobin Zhou, ${ }^{2}$ Yu Wu, ${ }^{1}$ You Yang, ${ }^{1}$ Wensheng Li, ${ }^{1}$ Jianhua Lu, ${ }^{1}$ and \\ Yunzhao $\mathrm{Hu}^{1}$ \\ ${ }^{1}$ Department of Cardiology, Shunde Hospital, Southern Medical University, Foshan, China \\ ${ }^{2}$ Department of Cardiology, Nanfang Hospital of Southern Medical University, Guangzhou 510515, China \\ Correspondence should be addressed to Jianhua Lu; lujianhuasums@126.com and Yunzhao Hu; huyunzhao4406@163.com
}

Received 10 September 2017; Accepted 20 September 2017; Published 4 October 2017

Academic Editor: Giuseppe Murdaca

Copyright (c) 2017 Yuli Huang et al. This is an open access article distributed under the Creative Commons Attribution License, which permits unrestricted use, distribution, and reproduction in any medium, provided the original work is properly cited.

\begin{abstract}
Background. Soluble urokinase plasminogen activator receptor (suPAR) is a novel marker of chronic inflammation and is considered to be a risk factor for coronary artery disease (CAD) in Caucasians. This study investigated the role of suPAR in young Chinese patients with CAD. Methods. The study involved a total of 196 consecutive young (age $\leq 55$ years) patients with angiographically proven CAD and 188 age-matched non-CAD individuals as controls. Traditional risk factors were evaluated using conventional assays, and levels of suPAR were measured by sandwich enzyme-linked immunosorbent assays. Results. Levels of suPAR were significantly correlated with age $(r=0.20, P=0.04)$, smoking $(r=0.33, P=0.008)$, body mass index $(r=0.21, P=0.03)$, and high-sensitivity C-reactive protein (hs-CRP; $r=0.31, P=0.01$ ). Multivariate logistic regression analysis showed that male sex (odds ratio $(\mathrm{OR})=3.12 ; 95 \%$ confidence interval $(\mathrm{CI})=1.18-8.25, P=0.02$ ), smoking $(\mathrm{OR}=3.41,95 \% \mathrm{CI}=1.55-7.50, P=0.002)$, triglyceride $(\mathrm{OR}=1.89,95 \% \mathrm{CI}=1.10-3.25, P=0.02)$, high-sensitivity C-reactive protein $(\mathrm{OR}=1.24,95 \% \mathrm{CI}=1.02-0.03, P=0.03)$, and suPAR $(\mathrm{OR}=1.37,95 \% \mathrm{CI}=1.09-1.72, P=0.007)$ were independently associated with CAD risk in young patients. Conclusions. SuPAR is a novel independent risk factor for CAD in young Chinese patients. Further studies evaluating the effect of anti-inflammatory treatment on the suPAR levels and the risk of CAD are needed.
\end{abstract}

\section{Introduction}

Coronary artery disease (CAD) is a major cause of mortality worldwide $[1,2]$. Although CAD is generally associated with older age, it has recently been reported more frequently in younger individuals [3]. It has been reported that nearly $23 \%$ of patients with acute coronary syndrome (ACS) are under 55 years old [4]. The prevalence of CAD in younger patients has become common in China during the past decade, causing a great burden on society and health care costs [5].

The risk factors for CAD in young patients are different from those in older patients [3]. Traditional risk factors, such as hypertension, diabetes mellitus (DM), and hypercholesterolemia, are less prevalent in young patients with CAD. A recent study of myocardial infarction patients showed that $36 \%$ of young patients had none, or only one, traditional risk factor for CAD [6]. These patients would have been classified as low risk according to existing risk scoring systems of cardiovascular disease (CVD), such as the Framingham Risk Score (FRS). Therefore, there is a need for identifying other novel CAD risk factors in young patients.

The urokinase plasminogen activator receptor (UPAR) is a three-domain membrane-bound receptor [7]. It is expressed on several immune cells, endothelial cells, and smooth muscle cells and is involved in several immune functions, including adhesion, migration, angiogenesis, fibrinolysis, and cell proliferation [8]. Cleavage of the membranebound UPAR during inflammatory stimulation releases the soluble form, suPAR, into the circulation. The level of suPAR has been considered as a marker of immune activation and low-grade inflammation [9]. Recent studies have shown that suPAR is associated with an increased risk of CVD and mortality in the general population [10-13]. Furthermore, 
the Danish MONICA10 (monitoring trends and determinants of cardiovascular disease) study showed that the association between elevated suPAR and increased risk of CVD was stronger in younger individuals [14]. However, most of the evidence was from the Caucasian populations and there have only been few reports of the association of suPAR with the risk of CAD in Chinese populations. Therefore, this study aimed to evaluate the association between levels of suPAR and the risk of CAD in young Chinese patients, after adjusting for traditional CAD risk factors and FRS.

\section{Materials and Methods}

2.1. Subjects. The study complied with the Declaration of Helsinki and was approved by the Ethics Committee of Shunde Hospital, Southern Medical University, China. Written informed consent was obtained from all participants. In accordance with the previous reports, young patients with CAD were defined as those presenting with initial CAD symptoms at $\leq 55$ years of age $[15,16]$. A total of 196 young patients diagnosed with CAD by coronary angiography (CAG) were enrolled between September 2012 and September 2015. CAG was performed using the standard Judkins technique through the femoral or radial artery with an Allura Xper FD20 (Philips, Amsterdam, Netherlands). CAD was defined as $\geq 50 \%$ stenosis of the lumen diameter in at least one major coronary artery, including the left main coronary artery, left anterior descending branch, left circumflex branch, and right coronary artery. Two independent interventional cardiologists evaluated coronary artery stenosis.

A total of 188 age-matched individuals with normal CAG or negative findings by ultrafast coronary computed tomography angiography (CCTA) were enrolled as controls. Coronary angiography or CCTA was performed because of chest discomfort with suspected ischemic findings, such as ST deviation in an electrocardiograph, regional dyskinesia by echocardiography, or difficulty in differential diagnosis. Patients with uncontrolled infectious diseases, autoimmune diseases, hormone replacement therapy after menopause, malignancy, severe renal dysfunction (creatinine $\geq 3 \mathrm{mg}$ / $\mathrm{dL}$ ), and psychiatric disorders were excluded.

2.2. Laboratory Measurements. Venous blood samples were collected after overnight fasting. Levels of fasting plasma glucose, total cholesterol (TC), triglyceride (TG), high-density lipoprotein cholesterol (HDL-C), and serum creatinine (SCr) were measured using an Olympus AU2700 automatic biochemical analyzer (Japan). Low-density lipoprotein cholesterol (LDL-C) was calculated using the Friedewald equation. We used the high-sensitivity nephelometric method to determine the levels of hs-CRP. After centrifugation at $1500 \times \mathrm{g}$ for $10 \mathrm{~min}$ at $4^{\circ} \mathrm{C}$, plasma samples were stored at $-80^{\circ} \mathrm{C}$ for future measurements of levels of suPAR. Levels of suPAR were measured using the commercial ELISA suPARnostic kit (ViroGates, Copenhagen, Denmark) according to the manufacturer's instructions. The interassay and intra-assay coefficients of variation were $6.2 \%$ and
$4.3 \%$, respectively. It has previously been shown that suPAR is highly stable throughout several freezing and thawing cycles [17].

2.3. Definition of Conventional Risk Factors for CAD. Conventional CAD risk factors evaluated in this study included the following: (1) Positive family history of premature $\mathrm{CAD}$ was defined as a diagnosis of CAD in a first-degree male relative $<55$ years old or first-degree female relative $<65$ years old. (2) With regard to smoking status, participants were classified as current smokers if they reported smoking regularly during the preceding year. Subjects who had never smoked or had stopped smoking at least 1 year before enrollment were classified as nonsmokers. (3) Hypertension was defined as per the Joint National Committee on Prevention, Detection, Evaluation, and Treatment of High Blood Pressure (JNC) VII guidelines [18]. (4) Diabetes mellitus was defined based on the 2003 American Diabetes Association criteria [19]. (5) Dyslipidemia was defined as per the 2007 Guidelines for Prevention and Treatment of Dyslipidemia in Adults in China, including $\mathrm{TC} \geq 200 \mathrm{mg} / \mathrm{dL}, \quad \mathrm{LDL}-\mathrm{C} \geq 130 \mathrm{mg} / \mathrm{dL}, \mathrm{HDL}-\mathrm{C}<40 \mathrm{mg} / \mathrm{dL}$, and/or $\mathrm{TG} \geq 150 \mathrm{mg} / \mathrm{dL}$, or a history of receiving antidyslipidemia agents [20]. (6) BMI was indicative of being overweight $\left(24-27.9 \mathrm{~kg} / \mathrm{m}^{2}\right)$ or obese $\left(\geq 28 \mathrm{~kg} / \mathrm{m}^{2}\right)$ as per Chinese criteria [21]. (7) The estimated glomerular filtration rate (eGFR) was calculated using the modified Modification of Diet in Renal Disease equation adapted for Chinese subjects, as eGFR $=186 \times \mathrm{Scr}^{-1.154} \times \mathrm{age}^{-0.203} \times$ 0.742 (female) $\times 1.233$ (Chinese) [22].

Based on the conventional risk factors for CAD, we also calculated the absolute 10 year CAD event risk scores based on the FRS system modified by the National Cholesterol Education Program Adult Treatment Panel III (NCEP ATP III) guidelines [23].

2.4. Statistical Analysis. All statistical analysis was performed using IBM SPSS Statistics for Windows, Version 22.0 (IBM Corp., Armonk, NY, USA). Continuous variables are presented as median (interquartile range) or mean (standard deviation) as appropriate. Categorical variables are expressed as percentages. Continuous variables were compared using the Mann-Whitney $U$ or Student's $t$-test after testing for normality using the KolmogorovSmirnov test. Categorical variables were compared using the chi-square or Fisher's exact test, as appropriate. Pearson correlation for normal variables was used to evaluate the associations between study parameters. Skewed variables were logarithmically transformed (suPAR and hs-CRP) and analyzed as a continuous variable per one standard deviation of the log values.

Multiple logistic regression analysis was performed to evaluate the risk factors for CAD. Age, smoking history, hypertension, DM, family history of CAD, BMI, TG, TC, HDL-C, LDL-C, hs-CRP, eGFR, and suPAR levels were set as independent variables in the regression model. Odds ratios (ORs) and 95\% confidence intervals (CIs) were calculated. $P$ values $<0.05$ were considered statistically significant. Furthermore, collinearity diagnostic statistics were performed 
TABLE 1: Demographic and clinical characteristics of CAD patients and controls.

\begin{tabular}{|c|c|c|c|}
\hline & CAD group $(n=196)$ & Control group $(n=188)$ & $P$ value \\
\hline Age (years) & $48.3 \pm 9.3$ & $49.8 \pm 10.4$ & 0.14 \\
\hline $\operatorname{Men}(n(\%))$ & $142(72.4 \%)$ & $92(48.9 \%)$ & $<0.0001$ \\
\hline CAD family history $(n(\%))$ & $15(7.7 \%)$ & $11(5.9 \%)$ & 0.55 \\
\hline Current smokers $(n(\%))$ & $76(38.8 \%)$ & $31(16.5 \%)$ & $<0.0001$ \\
\hline Hypertension $(n(\%))$ & $49(25.0 \%)$ & $35(18.6 \%)$ & 0.14 \\
\hline Systolic blood pressure (mmHg) & $124.1 \pm 12.5$ & $123.0 \pm 12.8$ & 0.39 \\
\hline Diastolic blood pressure (mmHg) & $73.2 \pm 9.1$ & $71.6 \pm 8.8$ & 0.08 \\
\hline Diabetes mellitus ( $n(\%))$ & $23(11.7 \%)$ & $17(9.0 \%)$ & 0.41 \\
\hline Fasting blood glucose (mg/dl) & $90.3 \pm 17.8$ & $92.3 \pm 16.7$ & 0.26 \\
\hline Dyslipidemia ( $n(\%))$ & $63(32.1 \%)$ & $41(21.8 \%)$ & 0.03 \\
\hline $\mathrm{TC}(\mathrm{mg} / \mathrm{dl})$ & $189.0 \pm 38.1$ & $184.7 \pm 39.5$ & 0.28 \\
\hline LDL-C (mg/dl) & $129.3 \pm 27.9$ & $125.1 \pm 26.0$ & 0.13 \\
\hline HDL-C (mg/dl) & $41.5 \pm 13.8$ & $43.3 \pm 13.5$ & 0.14 \\
\hline $\mathrm{TG}(\mathrm{mg} / \mathrm{dl})$ & $157.6 \pm 29.4$ & $146.5 \pm 28.2$ & 0.0002 \\
\hline Overweight/obesity (n (\%)) & $61(31.1 \%)$ & $40(21.3 \%)$ & 0.04 \\
\hline Body mass index $\left(\mathrm{kg} / \mathrm{m}^{2}\right)$ & $24.8 \pm 5.3$ & $23.7 \pm 4.6$ & 0.03 \\
\hline eGFR (mL/min/1.73 m2) & $111.2 \pm 35.0$ & $115.4 \pm 39.8$ & 0.27 \\
\hline hs-CRP (mg/dl) & $5.36(1.24-11.65)$ & $2.28(0.75-7.21)$ & $<0.0001$ \\
\hline $\operatorname{suPAR}(\mathrm{ng} / \mathrm{ml})$ & $5.8(1.3-11.8)$ & $2.8(1.0-6.5)$ & $<0.0001$ \\
\hline 10-year CAD event risk (\%) & $5.8 \pm 4.7$ & $5.0 \pm 4.0$ & 0.07 \\
\hline
\end{tabular}

Data are presented as mean $( \pm \mathrm{SD})$, median (interquartile range), or percentages. CAD: coronary artery disease; eGFR: estimated glomerular filtration rate; TC: total cholesterol; TG: triglyceride; HDL-C: high-density lipoprotein-cholesterol; LDL-C: low-density lipoprotein-cholesterol; hs-CRP: high-sensitivity C-reactive protein; suPAR: soluble urokinase plasminogen activator receptor.

to test the multicollinearity (strong correlations among independent variables). Variance inflation factor values $>2.5$ or tolerance $<0.4$ may indicate concern for multicollinearity in logistic regression models.

\section{Results}

3.1. Clinical Characteristics of Patients. We screened 214 young patients diagnosed with CAD by CAG and 203 agematched controls. Based on the predefined exclusion criteria, 17 patients and 15 controls were excluded (eight patients with CAD, five controls with severe renal dysfunction, two patients with CAD with severe hepatic insufficiency, six controls with a history of anxiety or depression, eight patients with $\mathrm{CAD}$, and four controls with uncontrolled infectious diseases). Therefore, 196 young patients with CAD (142 men, 54 women) and 188 age-matched controls (101 men, 87 women) were ultimately enrolled. Of the young patients diagnosed with CAD, 48 were classified as having unstable angina, 86 as having ST-segment elevation myocardial infarction, and 62 as having non-ST-segment elevation myocardial infarction. Table 1 shows the demographics and clinical characteristics of all participants.

Compared with the control group, the CAD group had a higher proportion of smoking, overweight/obesity, and dyslipidemia $(P<0.05)$, but the differences in the proportions of a positive family history of CAD, hypertension, and $\mathrm{DM}$ were not significant (all $P>0.05$ ). The levels of TG, hsCRP, and suPAR were significantly higher in CAD patients
TABLE 2: Correlation of suPAR and other cardiovascular risk factors.

\begin{tabular}{lcc}
\hline Variables & $r$ value & $P$ value \\
\hline Age & 0.20 & 0.04 \\
Sex & -0.11 & 0.21 \\
Smoking & 0.33 & 0.008 \\
SBP & 0.09 & 0.78 \\
DBP & 0.17 & 0.12 \\
FPG & -0.10 & 0.56 \\
TC & 0.15 & 0.11 \\
LDL-C & 0.19 & 0.09 \\
HDL-C & -0.14 & 0.08 \\
TG & 0.18 & 0.12 \\
BMI & 0.21 & 0.03 \\
eGFR & 0.15 & 0.26 \\
hs-CRP & 0.31 & 0.01 \\
\hline
\end{tabular}

suPAR and hs-CRP were skewed variables and logarithmically transformed. suPAR: soluble urokinase plasminogen activator receptor; SBP: systolic blood pressure; DBP: diastolic blood pressure; FPG: fasting blood glucose; TC: total cholesterol; LDL-C: low-density lipoprotein-cholesterol; HDL-C: high-density lipoprotein-cholesterol; TG: triglyceride; BMI: body mass index; eGFR: estimated glomerular filtration rate; hs-CRP: high-sensitivity C-reactive protein.

than in controls (all $p<0.05$ ), but there were no significant differences in other cardiovascular risk factors between the control group and the CAD group (all $p>0.05$ ) (Table 1$)$. 
TABLE 3: Risk factors for CAD in young patients in multivariate logistic regression analysis.

\begin{tabular}{|c|c|c|c|}
\hline Risk factors & OR & $95 \% \mathrm{CI}$ & $P$ value \\
\hline Sex (male versus female) & 3.12 & $1.18-8.25$ & 0.02 \\
\hline Age (per 10 years) & 1.32 & $0.85-2.05$ & 0.22 \\
\hline Smoking (yes versus no) & 3.41 & $1.55-7.50$ & 0.002 \\
\hline CAD family history (yes versus no) & 1.10 & $0.58-2.09$ & 0.77 \\
\hline BMI $\left(\geq 24 \mathrm{~kg} / \mathrm{m}^{2}\right.$ versus $\left.<24 \mathrm{~kg} / \mathrm{m}^{2}\right)$ & 1.34 & $0.97-1.85$ & 0.08 \\
\hline DM (yes versus no) & 1.21 & $0.82-1.76$ & 0.33 \\
\hline Hypertension (yes versus no) & 1.25 & $0.37-4.22$ & 0.72 \\
\hline $\mathrm{TG}(\geq 1.7 \mathrm{mmol} / \mathrm{L}$ versus $<1.7 \mathrm{mmol} / \mathrm{L})$ & 1.89 & $1.10-3.25$ & 0.02 \\
\hline $\mathrm{TC}(\geq 5.18 \mathrm{mmol} / \mathrm{L}$ versus $<5.18 \mathrm{mmol} / \mathrm{L})$ & 1.04 & $0.75-1.44$ & 0.81 \\
\hline LDL-C ( $\geq 3.37 \mathrm{mmol} / \mathrm{L}$ versus $<3.37 \mathrm{mmol} / \mathrm{L})$ & 1.17 & $0.88-1.56$ & 0.28 \\
\hline $\mathrm{HDL}-\mathrm{C}(\geq 1.04 \mathrm{mmol} / \mathrm{L}$ versus $<1.04 \mathrm{mmol} / \mathrm{L})$ & 0.97 & $0.12-7.84$ & 0.98 \\
\hline eGFR (per $10 \mathrm{~mL} / \mathrm{min} / 1.73 \mathrm{~m}^{2}$ ) & 1.58 & $0.42-5.94$ & 0.50 \\
\hline Log hs-CRP (per SD) & 1.24 & $1.02-1.51$ & 0.03 \\
\hline Log suPAR (per SD) & 1.37 & $1.09-1.72$ & 0.007 \\
\hline
\end{tabular}

BMI: body mass index; CAD: coronary artery disease; CHD: coronary heart disease; DM: diabetes mellitus; CI: confidence interval; eGFR: estimated glomerular filtration rate; HDL-C: high-density lipoprotein cholesterol; hs-CRP: high-sensitivity C-reactive protein; LDL-C: low-density lipoprotein cholesterol; OR: odds ratio; suPAR: soluble urokinase plasminogen activator receptor; TC: total cholesterol; TG: triglyceride.

3.2. Correlation between suPAR and Other Risk Factors for $C A D$. Table 2 shows correlations between suPAR and other clinical variables. Plasma levels of suPAR were significantly positively correlated with age $(r=0.20, P=0.04)$, smoking status $(r=0.33, P=0.008)$, BMI $(r=0.21, P=0.03)$, and hs-CRP levels $(r=0.31, P=0.01)$, but not with levels of FPG, SBP, DBP, TG, TC, HDL-C, LDL-C, or eGFR (all $P>0.05)$.

3.3. Levels of suPAR and Risk of CAD in Young Patients. Multivariate logistic regression analysis revealed that male sex $(\mathrm{OR}=3.12,95 \% \mathrm{CI}=1.18-8.25, \quad P=0.02)$, smoking $(\mathrm{OR}=3.41,95 \% \mathrm{CI}=1.55-7.50, P=0.002)$, TG $(\mathrm{OR}=1.89$, 95\% CI $=1.10-3.25, \quad P=0.02)$, hs-CRP $(\mathrm{OR}=1.24,95 \%$ $\mathrm{CI}=1.02-0.03, \quad P=0.03), \quad$ and $\operatorname{suPAR} \quad(\mathrm{OR}=1.37,95 \%$ $\mathrm{CI}=1.09-1.72, \quad P=0.007)$ were independently associated with CAD risk in young patients (Table 3 ). Multicollinearity analysis showed that the variance inflation factor value was $>2.5$, and the tolerance factor was $<0.4$, which indicates that there was no obvious multicollinearity in the logistic regression models.

\section{Discussion}

To our knowledge, this is the first report of suPAR being an independent risk factor for CAD in young Chinese patients. This correlation was observed even after adjustment for traditional cardiovascular risk factors, including hypertension, smoking, DM, dyslipidemia, and hs-CRP levels.

The immune system plays an important role in the pathogenesis of atherosclerosis. Chronic low-grade inflammation is significantly associated with the risk of CVD, while hs-CRP is the most common recommended risk marker of inflammation in cardiovascular risk stratification and has been widely evaluated in epidemiological studies [24]. Our results indicate that levels of the novel inflammatory marker, suPAR, are positively correlated with hs-CRP levels. However, the association between suPAR and the risk of CAD in young Chinese patients is independent of hs-CRP levels. This is in agreement with a previous report by Ghasemzedah et al. who showed that suPAR, hs-CRP, fibrin degradation product, and heat shock protein-70 are independent risk factors for CAD [25]. Lyngbaek et al. have shown that CRP is positively associated with anthropometric measures, such as BMI and waist circumference, whereas suPAR is linked to endothelial dysfunction and atherosclerosis [26]. Taken together, the above results indicate that these biomarkers may each be involved in the activation of different pathophysiological pathways leading to CAD.

Compared with hs-CRP, suPAR may be a more reliable factor for evaluating the risk of CAD. First, the role of hs-CRP in inflammation is controversial and it has been suggested that hs-CRP is merely a passive risk marker rather than a risk factor $[13,27]$. For example, a Mendelian randomization study indicated that hs-CRP is a risk marker and not a causal factor for ischemic vascular disease [28]. In contrast, many in vivo and in vitro studies have shown that suPAR is involved in the development of atherosclerosis $[29,30]$. Second, CRP is a highly inducible acute-phase protein, whereas suPAR does not show major variation. Levels of suPAR showed an increase of $15 \%$ following acute myocardial infarction, compared with a $365 \%$ increase in hs-CRP levels [31]. Furthermore, suPAR levels have been shown to have minimal circadian variation, which makes it a more suitable candidate as a clinical biomarker [14].

The exact pathophysiological mechanisms underlying the association between suPAR and CAD are still unclear, but we suggest several possibilities. First, suPAR and its ligand are involved in many pathogenic pathways, including pericellular proteolysis and matrix degradation, plasminogen activation and fibrinolysis, cell adhesion, migration, and proliferation [32]. Second, the suPAR molecule has intrinsic 
chemotactic properties and modulates the ability of monocytes to migrate in response to other chemokines [33]. Third, suPAR may also be involved in the rupture of atherosclerotic plaques, which causes acute attacks of CAD [12].

It has previously been reported that lifestyle modification, such as smoking cessation, and weight loss, would lower the levels of plasma suPAR $[34,35]$. However, our study found that the association between increased levels of suPAR and the risk of CAD in young patients could not be completely explained by traditional cardiovascular risk factors, including smoking and obesity. Therefore, other measures to lower suPAR levels and further lower the risk of CAD should be explored. Anti-inflammatory treatment with canakinumab, a therapeutic monoclonal antibody targeting interleukin-1 $\beta$, led to a significantly lower rate of recurrent CVD than placebo [36]. It has been reported that suPAR can be released from endothelial cells following stimulation by interleukin-1 $\beta$ [37]. It is, however, unclear if the observed canakinumab's effect on CVD is mediated by lowering the levels of suPAR.

The current study has some limitations. First, the casecontrol design of the study could not definitely evaluate a potential risk factor since reverse causality may exist. However, suPAR levels have been shown to be within minimal variation after acute myocardial infarction [31], and hence reverse causality is limited. Second, most of the controls were defined as non-CAD by CCTA, but not CAG, because of the excellent negative predictive value of CCTA in the diagnosis of CAD [38].

\section{Conclusions}

We report that suPAR, a novel inflammatory marker, is positively associated with the risk of CAD in young Chinese patients. Further studies to evaluate the effect of antiinflammatory treatment on suPAR levels and the risk of CAD are needed.

\section{Ethical Approval}

The study complied with the Declaration of Helsinki and was approved by the Ethics Committee of Shunde Hospital, Southern Medical University. Written informed consent was obtained from all participants.

\section{Disclosure}

The funding source had no role in the study design, data collection, data analysis, data interpretation, or writing of the article.

\section{Conflicts of Interest}

The authors declare that they have no competing interests.

\section{Authors' Contributions}

Yuli Huang is the first author and performed the data collection, data analysis, and manuscript preparation. Haobin Zhou and Yu Wu performed the data analysis and manuscript preparation. You Yang and Wensheng Li performed the data collection. Yuli Huang and Jianhua $\mathrm{Lu}$ are the principle investigators and are responsible for the study design, data collection, data analysis, and manuscript preparation. The manuscript was approved by all above authors.

\section{Acknowledgments}

This work is supported by the Key Specialist Department Training Project of Foshan City, Guangdong, China (FSPY3-2015034).

\section{References}

[1] G. N. Levine, E. R. Bates, J. A. Bittl et al., "2016 ACC/AHA guideline focused update on duration of dual antiplatelet therapy in patients with coronary artery disease: a report of the American College of Cardiology/American Heart Association Task Force on clinical practice guidelines," Journal of the American College of Cardiology, vol. 68, pp. 1082-1115, 2016.

[2] J. Li, X. Li, Q. Wang et al., "ST-segment elevation myocardial infarction in China from 2001 to 2011 (the China PEACERetrospective Acute Myocardial Infarction Study): a retrospective analysis of hospital data," Lancet, vol. 385, pp. 441-451, 2015.

[3] N. Shah, A. M. Kelly, N. Cox, C. Wong, and K. Soon, "Myocardial infarction in the "young": risk factors, presentation, management and prognosis," Heart, Lung \& Circulation, vol. 25, pp. 955-960, 2016.

[4] A. Rosengren, L. Wallentin, M. Simoons et al., "Age, clinical presentation, and outcome of acute coronary syndromes in the Euroheart acute coronary syndrome survey," European Heart Journal, vol. 27, pp. 789-795, 2006.

[5] X. Wang, M. Gao, S. Zhou et al., "Trend in young coronary artery disease in China from 2010 to 2014: a retrospective study of young patients $\leq 45$," BMC Cardiovascular Disorders, vol. 17, p. 18, 2017.

[6] K. Matsis, A. Holley, A. Al-Sinan, P. Matsis, P. D. Larsen, and S. A. Harding, "Differing clinical characteristics between young and older patients presenting with myocardial infarction,” Heart, Lung \& Circulation, vol. 26, pp. 566-571, 2017.

[7] Q. Huai, A. P. Mazar, A. Kuo et al., "Structure of human urokinase plasminogen activator in complex with its receptor," Science, vol. 311, pp. 656-659, 2006.

[8] B. Fuhrman, "The urokinase system in the pathogenesis of atherosclerosis," Atherosclerosis, vol. 222, pp. 8-14, 2012.

[9] T. H. Haupt, T. Kallemose, S. Ladelund et al., "Risk factors associated with serum levels of the inflammatory biomarker soluble urokinase plasminogen activator receptor in a general population," Biomarker Insights, vol. 9, pp. 91-100, 2014.

[10] S. Botha, C. M. Fourie, R. Schutte, J. Eugen-Olsen, R. Pretorius, and A. E. Schutte, "Soluble urokinase plasminogen activator receptor as a prognostic marker of all-cause and cardiovascular mortality in a black population," International Journal of Cardiology, vol. 184, pp. 631-636, 2015.

[11] D. J. Eapen, P. Manocha, N. Ghasemzadeh et al., "Soluble urokinase plasminogen activator receptor level is an independent predictor of the presence and severity of coronary artery disease and of future adverse events," Journal of the American Heart Association, vol. 3, article e1118, 2014. 
[12] M. Persson, G. Ostling, G. Smith et al., "Soluble urokinase plasminogen activator receptor: a risk factor for carotid plaque, stroke, and coronary artery disease," Stroke, vol. 45, pp. 18-23, 2014.

[13] M. H. Sorensen, O. Gerke, J. Eugen-Olsen et al., "Soluble urokinase plasminogen activator receptor is in contrast to high-sensitive C-reactive-protein associated with coronary artery calcifications in healthy middle-aged subjects," Atherosclerosis, vol. 237, pp. 60-66, 2014.

[14] J. Eugen-Olsen, O. Andersen, A. Linneberg et al., "Circulating soluble urokinase plasminogen activator receptor predicts cancer, cardiovascular disease, diabetes and mortality in the general population," Journal of Internal Medicine, vol. 268, pp. 296-308, 2010.

[15] R. Pelletier, N. A. Khan, J. Cox et al., "Sex versus genderrelated characteristics: which predicts outcome after acute coronary syndrome in the young?," Journal of the American College of Cardiology, vol. 67, pp. 127-135, 2016.

[16] Y. Yang, M. Bi, L. Xiao et al., "Perceived stress status and sympathetic nervous system activation in young male patients with coronary artery disease in China," European Journal of Internal Medicine, vol. 26, pp. 726-730, 2015.

[17] S. Lyngbaek, J. L. Marott, T. Sehestedt et al., "Cardiovascular risk prediction in the general population with use of suPAR, CRP, and Framingham risk score," International Journal of Cardiology, vol. 167, pp. 2904-2911, 2013.

[18] A. V. Chobanian, G. L. Bakris, H. R. Black et al., "Seventh report of the joint national committee on prevention, detection, evaluation, and treatment of high blood pressure," Hypertension, vol. 42, pp. 1206-1252, 2003.

[19] Expert Committee on the Diagnosis and Classification of Diabetes Mellitus, "Report of the expert committee on the diagnosis and classification of diabetes mellitus," Diabetes Care, vol. 26, Supplement 1, pp. S5-S20, 2003.

[20] Joint Committee for Developing Chinese guidelines on Prevention and Treatment of Dyslipidemia in Adults, "Chinese guidelines on prevention and treatment of dyslipidemia in adults," Zhonghua Xin Xue Guan Bing Za Zhi, vol. 35, pp. 390-419, 2007.

[21] H. Cui, F. Wang, L. Fan et al., "Association factors of target organ damage: analysis of 17,682 elderly hypertensive patients in China," Chinese Medical Journal, vol. 124, pp. 3676-3681, 2011.

[22] Y. C. Ma, L. Zuo, J. H. Chen et al., "Modified glomerular filtration rate estimating equation for Chinese patients with chronic kidney disease," Journal of the American Society of Nephrology, vol. 17, no. 10, pp. 2937-2944, 2006.

[23] R. L. Talbert, "New therapeutic options in the National Cholesterol Education Program Adult Treatment Panel III," The American Journal of Managed Care, vol. 8, pp. S301S307, 2002.

[24] T. A. Pearson, G. A. Mensah, Y. Hong, and S. J. Smith, "CDC/AHA Workshop on markers of inflammation and cardiovascular disease: application to clinical and public health practice: overview," Circulation, vol. 110, pp. e543e544, 2004.

[25] N. Ghasemzedah, S. S. Hayek, Y. A. Ko et al., "Pathway-specific aggregate biomarker risk score is associated with burden of coronary artery disease and predicts near-term risk of myocardial infarction and death," Circulation. Cardiovascular Quality and Outcomes, vol. 10, 2017.
[26] S. Lyngbaek, T. Sehestedt, J. L. Marott et al., "CRP and suPAR are differently related to anthropometry and subclinical organ damage," International Journal of Cardiology, vol. 167, pp. 781-785, 2013.

[27] T. Shah, J. P. Casas, J. A. Cooper et al., "Critical appraisal of CRP measurement for the prediction of coronary heart disease events: new data and systematic review of 31 prospective cohorts," International Journal of Epidemiology, vol. 38, pp. 217-231, 2009.

[28] J. Zacho, A. Tybjaerg-Hansen, J. S. Jensen, P. Grande, H. Sillesen, and B. G. Nordestgaard, "Genetically elevated Creactive protein and ischemic vascular disease," The New England Journal of Medicine, vol. 359, pp. 1897-1908, 2008.

[29] A. E. Cozen, H. Moriwaki, M. Kremen et al., "Macrophagetargeted overexpression of urokinase causes accelerated atherosclerosis, coronary artery occlusions, and premature death," Circulation, vol. 109, pp. 2129-2135, 2004.

[30] T. Sehestedt, S. Lyngbaek, J. Eugen-Olsen et al., "Soluble urokinase plasminogen activator receptor is associated with subclinical organ damage and cardiovascular events," Atherosclerosis, vol. 216, pp. 237-243, 2011.

[31] S. Lyngbaek, J. L. Marott, D. V. Moller et al., "Usefulness of soluble urokinase plasminogen activator receptor to predict repeat myocardial infarction and mortality in patients with ST-segment elevation myocardial infarction undergoing primary percutaneous intervention," The American Journal of Cardiology, vol. 110, pp. 1756-1763, 2012.

[32] F. Blasi and P. Carmeliet, "uPAR: a versatile signalling orchestrator," Nature Reviews Molecular Cell Biology, vol. 3, pp. 932-943, 2002.

[33] A. Edsfeldt, M. Nitulescu, H. Grufman et al., "Soluble urokinase plasminogen activator receptor is associated with inflammation in the vulnerable human atherosclerotic plaque," Stroke, vol. 43, pp. 3305-3312, 2012.

[34] J. Eugen-Olsen, S. Ladelund, and L. T. Sorensen, "Plasma suPAR is lowered by smoking cessation: a randomized controlled study," European Journal of Clinical Investigation, vol. 46, pp. 305-311, 2016.

[35] L. R. Pedersen, R. H. Olsen, C. Anholm et al., "Weight loss is superior to exercise in improving the atherogenic lipid profile in a sedentary, overweight population with stable coronary artery disease: a randomized trial," Atherosclerosis, vol. 246, pp. 221-228, 2016.

[36] P. M. Ridker, B. M. Everett, T. Thuren et al., "Antiinflammatory therapy with canakinumab for atherosclerotic disease," The New England Journal of Medicine, vol. 377, no. 12, pp. 1119-1131, 2017.

[37] T. Chavakis, A. K. Willuweit, F. Lupu, K. T. Preissner, and S. M. Kanse, "Release of soluble urokinase receptor from vascular cells," Thrombosis and Haemostasis, vol. 86, pp. 686693, 2001.

[38] M. S. Bittencourt, E. A. Hulten, V. Veeranna, and R. Blankstein, "Coronary computed tomography angiography in the evaluation of chest pain of suspected cardiac origin," Circulation, vol. 133, pp. 1963-1968, 2016. 


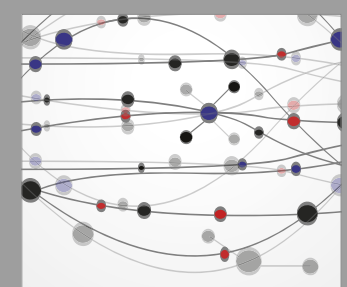

The Scientific World Journal
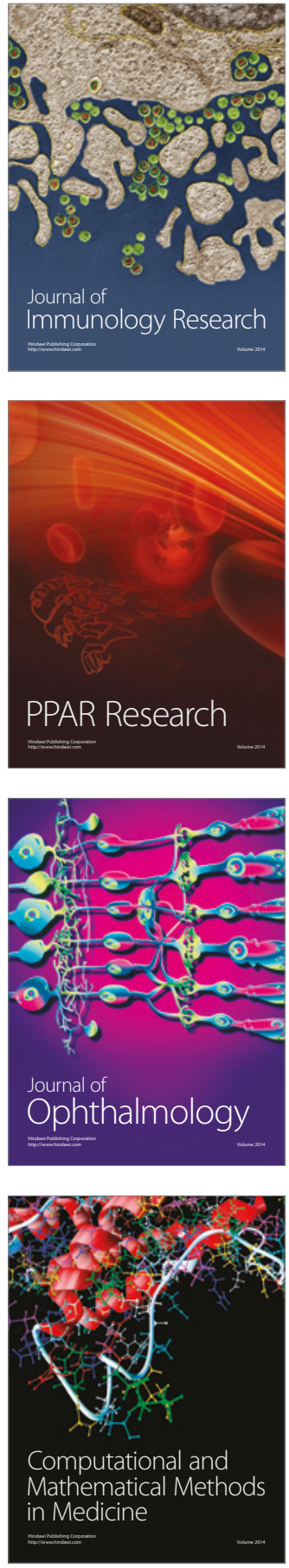

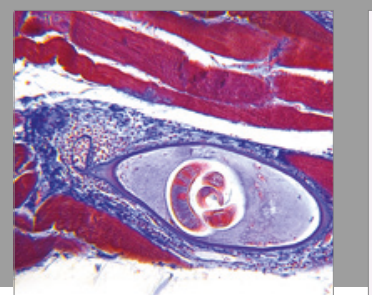

Gastroenterology Research and Practice
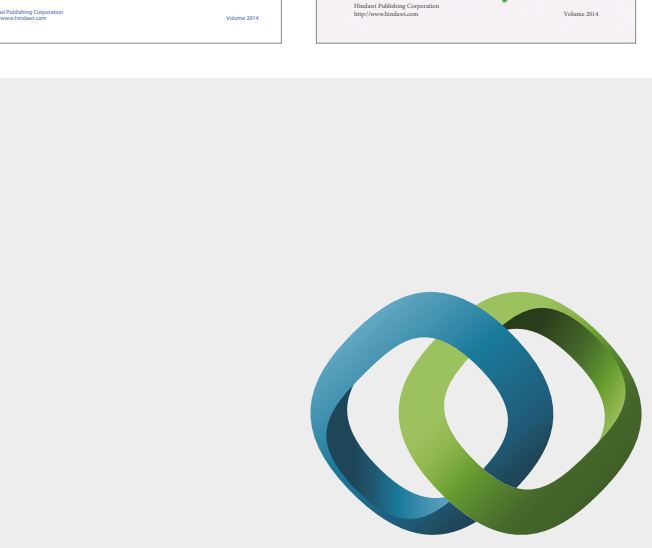

\section{Hindawi}

Submit your manuscripts at

https://www.hindawi.com
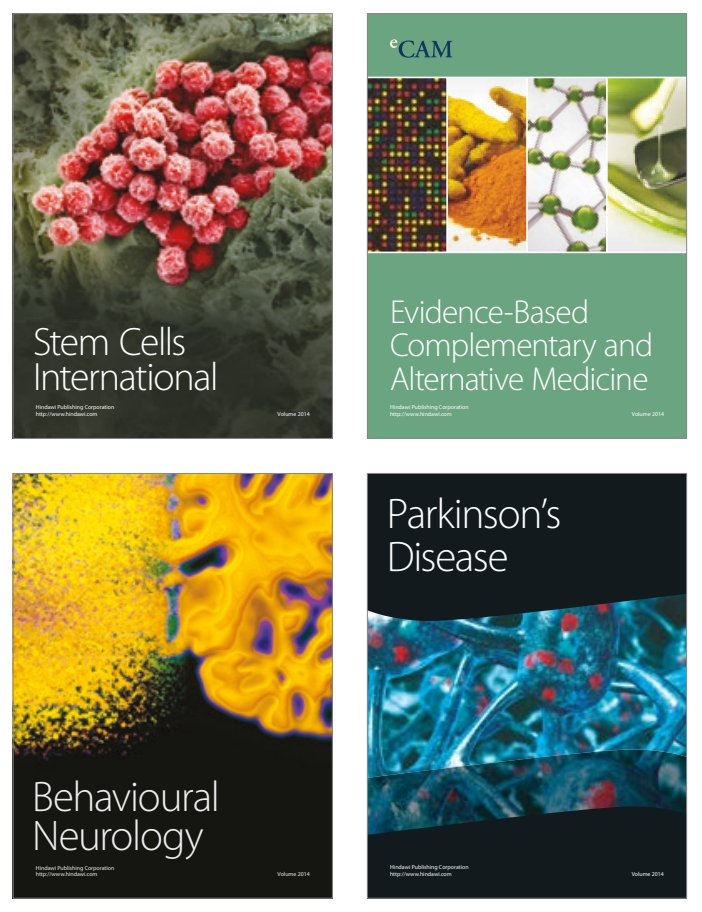
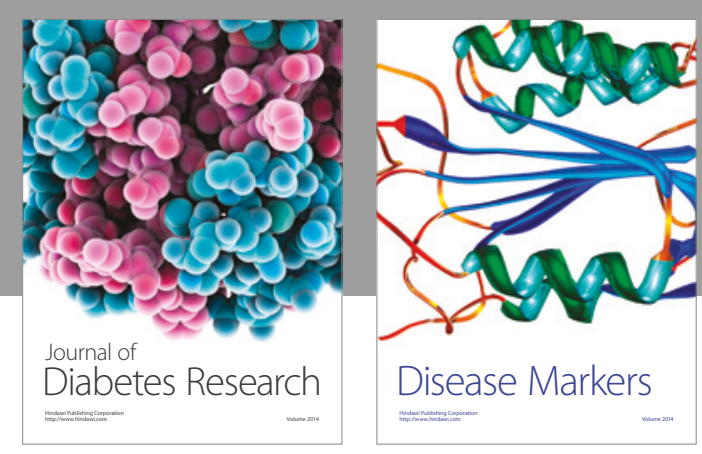

Disease Markers
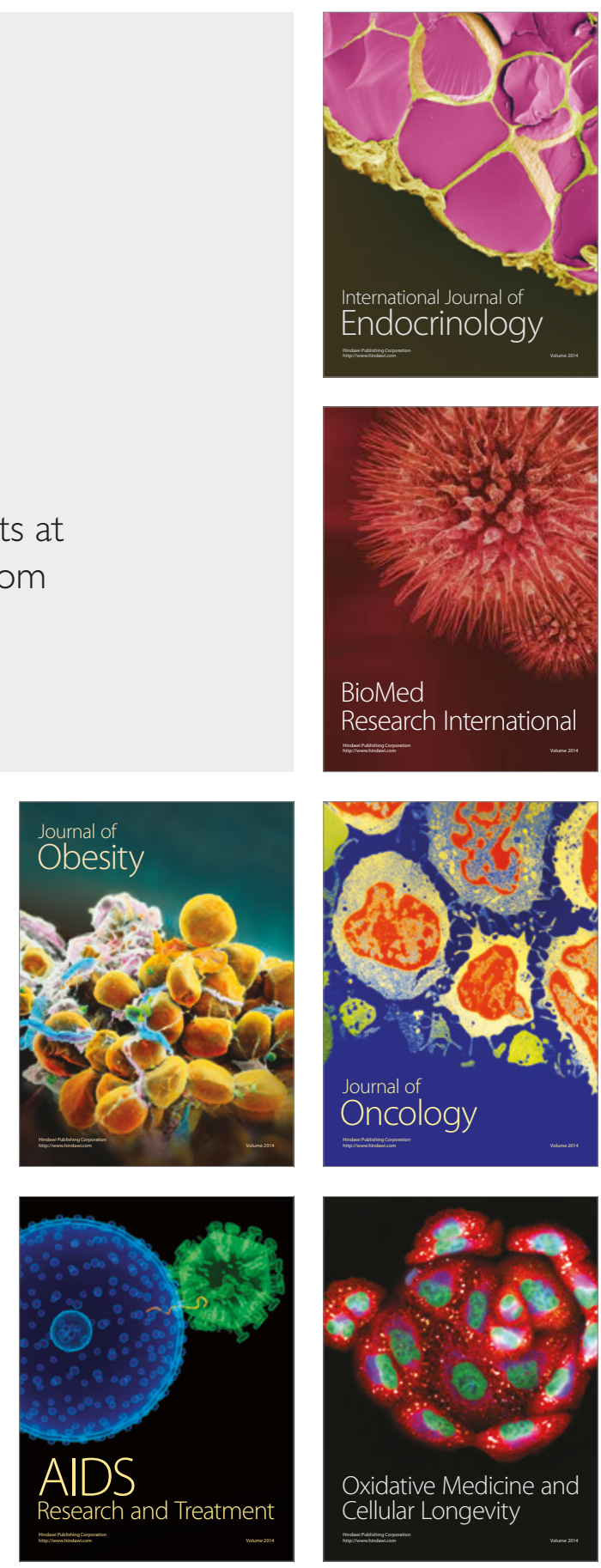